\title{
A constructivist theory of plane curves
}

by

\section{R. L. Goodste in (Leicester)}

Introduction. This paper develops a theory of $p$-curves, which are finite matrices with binary fraction elements. Roughly spealking, a $p$-curve is a finite assemblage of points in serial order on a grid, the jump from one point to the next being of fixed amount in one or other of two "directions". The concept of a plane curve is then introduced in terms of sequences of $p$-curves. The emphasis throughout the paper is on the strictly finitist character of the proof processes.

The present work on analysis situs is a preliminary to a study of curvilinear integrals.

Definitions. We denote integers by $i, j, k, l, m, n, \mu, v, p, q, r, s$, $t, \varrho, \sigma, \tau$ with or without suffixes, and binary fractions $m / 2^{p}$ by $a, b, c$, $d, x, y, \xi, \eta$ with or without suffixes or affixes; more specifically, for a given $p$ we write $x^{p}$, ete., for $m / 2^{p}$. The ordered pair $(x, y)$ is called a point, and the ordered pair $\left\langle x_{1}, y_{2}\right\rangle$ an interval; the ordered pair of intervals $\left\langle x_{1}, x_{2}\right\rangle\left\langle y_{1}, y_{2}\right\rangle$ (where $x_{1}<x_{2}, y_{1}<y_{2}$ ) is called a reotangle with vertices $\left(x_{r}, y_{s}\right), r=1,2$ and $s=1,2$. If

$$
2^{p} a_{r}^{p}, \quad 0 \leqslant r \leqslant \mu_{p}, \quad 2^{p} b_{s}^{p}, \quad 0 \leqslant s \leqslant v_{p}
$$

are the integers from $2^{p} x_{1}^{p}$ to $2^{p} x_{2}^{p}$ and from $2^{p} y_{1}^{p}$ to $2^{p} y_{2}^{p}$, respectively, then the points

$$
\left(a_{r}^{p}, b_{s}^{p}\right), \quad 0 \leqslant r \leqslant \mu_{p}, \quad 0 \leqslant \varepsilon \leqslant v_{p},
$$

are called the lattice points of the network

$$
F_{p}\left(\begin{array}{ll}
x_{1}^{p} & x_{2}^{p} \\
y_{1}^{p} & y_{2}^{p}
\end{array}\right)
$$

in the rectangle $\left\langle x_{1}^{p}, x_{2}^{p}\right\rangle\left\langle y_{1}^{p}, y_{2}^{p}\right\rangle$; the rectangles $\left\langle a_{r}^{p}, a_{r+1}^{p}\right\rangle\left\langle b_{s}^{p}, b_{s+1}^{p}\right\rangle, 0 \leqslant r \leqslant \mu_{n}$, $0 \leqslant s \leqslant v_{p}$ are called the $p$-eells of the rectangle $\left\langle x_{1}^{p}, x_{2}^{p}\right\rangle\left\langle y_{1}^{p}, y_{2}^{p}\right\rangle$ or of the network

$$
F_{p}\left(\begin{array}{ll}
x_{1}^{p} & x_{2}^{p} \\
y_{1}^{p} & y_{2}^{p}
\end{array}\right)
$$


For a given $k \geqslant 1$ the integers $i_{r}, j_{r}$ satisfy the equation

$$
\left|i_{r+1}-i_{r}\right|+\left|j_{r+1}-j_{r}\right|=1
$$

for all $r, 0 \leqslant r \leqslant k-1$, and $x_{r}^{p}=i_{r} / 2^{p}, y_{r}^{p}=j_{r} / 2^{p}$; then the ordered set of points

$$
\left(x_{r}^{p}, y_{r}^{p}\right), \quad 0 \leqslant r \leqslant k,
$$

is called a plane curve, or specifically a plane p-curve, joining the points $\left(x_{0}^{p}, y_{0}^{p}\right)$ and $\left(x_{k}^{p}, y_{k}^{p}\right)$. If

$$
\left|i_{r}-i_{s}\right|+\left|j_{r}-j_{s}\right|>0
$$

for all $r, s$ satisfying $0 \leqslant r<s \leqslant k$, then the curve $(0.2)$ is said to be simple and open.

(0.31) If $i_{r}, j_{r}$ satisfy the condition (0.3) for all $r, s$ such that $0 \leqslant r \leqslant s \leqslant k-1$ or $1 \leqslant r<s<k$, and if in addition, $i_{k}=i_{0}, j_{k}=j_{0}, k>4$, then the curve (0.2) is said to be simple and olosed.

If $x_{r}, y_{r}$ are periodic, with period $k$, and if $\left(x_{r}, y_{r}\right), 0 \leqslant r \leqslant k$, is a simple closed curve, then eo ipso the curve $\left(x_{r}, y_{r}\right), m \leqslant r \leqslant m+k$, is closed and simple. The curves $\left(x_{r}, y_{r}\right), 0 \leqslant r \leqslant k$, and $\left(x_{r}, y_{r}\right), m \leqslant r \leqslant m+k$ are said to be equivalent and mutually interchangeable.

If

and

$$
\begin{array}{ll}
i_{2 r}^{p+1}=2 i_{r}^{p}, & i_{2 r+1}^{p+1}=i_{r}^{p}+i_{r+1}^{p}, \\
j_{2 r}^{p+1}=2 j_{r}^{p}, & j_{2 r+1}^{p+1}=j_{r}^{p}+j_{r+1}^{p},
\end{array}
$$

where $i_{r}^{p}, j_{r}^{p}$ satisfy $(0.1)$, then obviously $i_{r}^{p+1}, j_{r}^{p+1}$ satisfy (0.1). The $p$-curve $\left(x_{r}^{p}, y_{r}^{p}\right), \quad 0 \leqslant r \leqslant k$, and the $(p+1)$-curve $\left(x_{r}^{p+1}, y_{r}^{p+1}\right), \quad 0 \leqslant r \leqslant 2 k$, (where $x_{r}^{q}=i_{r}^{q} / 2^{q}, y_{r}^{q}=j_{r}^{q} / 2^{q}$ for $\left.q=p, p+1\right)$ are said to be equivalent and mutually interchangeable.

We shall denote by $\xi$ (with one or more suffixes) a value taken by one or more of the numbers $x_{r}, 0 \leqslant r \leqslant k$, and by $\eta$ a value taken by one or more of $y_{r}, 0 \leqslant \dot{r} \leqslant k$. In a network $F_{p}, \xi+2^{-p}$ will be called the $s u c$ cessor of $\xi$ and denoted by $\xi^{\prime}$, and $\xi-2^{-p}$ will be called the predecessor of $\xi$ and denoted by $\xi^{*}$. Similarly we define $\eta^{\prime}, \eta^{*}$. The successor of $x_{r}$ of course is $x_{r+1}, r<k$, and the predecessor of $x_{r+1}$ is $x_{r}, r \geqslant 0$; we shall also call $x_{1}$ the successor of $x_{k}\left(=x_{0}\right)$ in a closed curve, and $x_{k-1}$ the predecessor of $x_{0}$. Similarly the successor of $y_{k}$ and the predecessor of $y_{0}$ are $y_{1}$ and $y_{k-1}$ respectively, when the curve is closed.

Such a pair as $\xi, \xi^{\prime}$ will be called a vertical strip, and such as $\eta, \eta^{\prime}$ a horizontal strip.

If $r_{n}, 0 \leqslant n \leqslant \mu$, are all the suffixes $r$ such that, either

$$
x_{r}=\xi, \quad x_{r+1}=\xi^{\prime} \quad \text { or } \quad x_{r}=\xi^{\prime}, \quad x_{r+1}=\xi
$$

in a simple closed curve $\left(x_{r}, y_{r}\right), 0 \leqslant r \leqslant l$, then the values $\eta_{n}$ of $y_{r_{n}}$, $0 \leqslant n \leqslant \mu$, are called the boundary levels in the vertical strip $\xi, \xi^{\prime}$ (note that $y_{r}=y_{r+1}$ since $\left.x_{r} \neq x_{r+1}\right)$. The $\eta^{\prime}$ s are all different since the points $\left(x_{n}, \eta_{n}\right),\left(x_{n+1}, \eta_{n}\right)$ cannot both occur twice in the set $\left(x_{r}, y_{i}\right), 0 \leqslant r \leqslant k$. Similarly we define the boundary levels in the horizontal strip $\eta, \eta^{\prime}$.

The foregoing definitions, and the proofs which follow, may be regarded as definition and proof sohemata formalisable by replacing the unspecified numbers and functions introduced by definite numbers and functions. The definitions, however, are also susceptible of formalisation in a free variable calculus.

1. ThEOREM 1. If $\left(x_{r}, y_{r}\right), 0 \leqslant r \leqslant k$, is a simple ourve, and if for some $m, n$ (where $0 \leqslant m<n \leqslant k$ if the curve is open, and $0 \leqslant m<n<k$ or $0<m \leqslant n<7$ if the ourve is elosed), and for all $s, t$ salisfying $m \leqslant s<t \leqslant n$, we have $y_{s}=y_{t}$, then the sequence $x_{r}, m \leqslant r \leqslant n$, is strictly monotonic.

We may suppose $n>m+2$, else there is nothing to prove. Let $x_{s}=\xi$ so that, since $y_{s+1}^{\prime}=y_{s}, x_{s+1}$ must be either $\xi^{\prime}$ or $\xi^{*}$; suppose the former, then since $x_{s+2}$ differs from both $x_{s}$ and $x_{s+1}$, and the values of $x_{s+1}, x_{s+2}$ are consecutive in $F_{p}$, therefore $x_{s+2}=\xi^{\prime \prime}$. Similarly, if $x_{s+1}=\xi^{*}$ then $x_{s+2}=\xi^{* *}$, and so, since $x_{m} \gtrless x_{m+1}$, the sequence $x_{r}, m \leqslant r \leqslant n$, is strictly monotonic.

2. THEOREM 2. If the integers $i_{r}$ satisfy the equation $(0.1)$, and if $0 \leqslant m<n \leqslant k$, then $i_{r}$ takes every integral value between $i_{m}$ and $i_{n}$ for a value of $r$ between $m$ and $n$.

For if $i_{m}<v<i_{n}$ and if $\mu$ is the smallest integer, greater than $m$, such that $i_{\mu} \geqslant v$, then $i_{\mu-1} \leqslant v-1$; but

$$
0 \leqslant\left(i_{\mu}-v\right)+\left(v-1-i_{\mu-1}\right)=\left(i_{\mu}-i_{\mu-1}\right)-1<0,
$$

and so $i_{\mu}=v$.

It follows that if $\left(x_{r}, y_{r}\right), 0 \leqslant r \leqslant k$, is a $p$-curve then $x_{r}$ attains every value $l / 2^{p}$ between $x_{m}, x_{n}$ for an $r$ between $m, n$ and $y_{r}$ attains every value $l / 2^{p}$ between $y_{m}, y_{n}$ for an $r$ between $m, n$.

3. Theorem 3. If the integers $i_{r}, 0 \leqslant r \leqslant k$, satisfy $(0.1)$, and $i_{k}=i_{0}$, and if for some $m, v, i_{0}<v, i_{m}=v$, then if $\lambda<k$ is the greatest suffix such that $i_{\lambda}=v$, we have $i_{\lambda+1}=v-1$; for otherwise $i_{\lambda+1}=v+1$, and by Theorem 2 we should have $i_{r}=v$ for some $r$, where $\lambda+1<r<k$. Similarly, if $i_{0}>v$ and $\mu$ is the least suffix for which $i_{\mu}=v$, then $i_{\mu-1}=v+1$.

4. THEorem 4. If $\left(x_{r}, y_{r}\right), 0 \leqslant r \leqslant k$, is a simple olosed curve; and if for some $m, n$

$$
x_{m}=\xi, \quad x_{m+1}=\xi^{\prime} \quad \text { and } \quad x_{n}=\xi^{\prime}, \quad x_{n+1}=\xi,
$$

then $m \neq n+1$ and $n \neq m+1$.

For by $0.31, x_{m+2} \geq \xi^{\prime}>x_{n+1}$ and $x_{n+2} \leqslant \xi<x_{m+1}$. 
5. THEOREM 5. If $\left(x_{r}, y_{r}\right), 0 \leqslant r \leqslant 7$, is a simple closed curve, then each pair of values $\xi, \xi^{\prime}$ is taken by consecutive $x$ 's an even number of times.

Proof. If there is no value of $r, 0 \leqslant r \leqslant k$, such that for a given pair $\xi, \xi^{\prime}, x_{r}=\xi$ and $x_{r+1}=\xi^{\prime}$ or $x_{r}=\xi^{\prime}$ and $x_{r+1}=\xi$, then the theorem is proved.

If there is a unique value of $r, 0 \leqslant r \leqslant k$, such that $x_{r}=\xi, x_{r+1}=\xi^{r}$ then there is an $s, 0 \leqslant s \leqslant k$, such that $x_{s}=\xi^{\prime}, x_{s+1}=\xi$. For if $x_{0}<\xi^{\prime}$ and $s$ is the greatest suffix such that $x_{s}=\xi^{\prime}$ then, by Theorem $3, x_{s+1}=\xi$. Moreover, by Theorem $4, s>r+1$.

If there is more than one value of $r$ for which $x_{r}=\xi, x_{r+1}=\xi^{\prime}$ then between any consecutive two such values, $m$ and $n$ say, there is a $\varrho$ such that

$$
x_{e}=\xi^{\prime}, \quad x_{e+1}=\xi, \quad \text { and } \quad \varrho+1<n .
$$

For if $\varrho+1$ is the least suffix between $m+1$ and $n$ such that $x_{Q+1}=\xi$ then, by Theorem $3, x_{e}=\xi^{\prime}$ (and $m+1<\varrho<n-1$, by Theorem 4). Similarly, between consecutive values of $r$ for which $x_{r}=\xi^{\prime}, x_{r+1}=\xi$ there is a value of $r$ for which $x_{r}=\xi, x_{r+1}=\xi^{\prime}$.

Let $m_{s}, 0 \leqslant s \leqslant \sigma$, where $m_{s}<m_{s+1}$, be all the values of $r, 0 \leqslant r \leqslant \pi$, for which $x_{r}=\xi, x_{r+1}=\xi^{\prime}$, and let $\mu_{t}, 0 \leqslant t \leqslant \tau$, where $\mu_{t}<\mu_{t+1}$ be all the values of $r, 0 \leqslant r \leqslant k$, for which $x_{r}=\xi^{\prime}, x_{r+1}=\xi$.

We may without loss of generality suppose that $m_{0}<\mu_{0}$. Since there is a $\mu$ between $m_{0}$ and $m_{x}$ therefore $\mu_{0}$ lies between $m_{0}$ and $m_{1}$, and since there is an $m$ between $\mu_{0}$ and $\mu_{1}$, therefore $m_{1}$ lies between $\mu_{0}$ and $\mu_{1}$, and so $\mu_{0}$ is the only $\mu$ between $m_{0}$ and $m_{1}$.

Similarly $\mu_{s}$ is the only $\mu$ between $m_{s}$ and $m_{s+1}, 0 \leqslant s \leqslant \sigma-1$. By considering the equivalent closed ourve $\left(x_{r}, y_{r}\right), m_{\sigma} \leqslant r \leqslant m_{\sigma}+k$, it follows that there is a unique $\tau$ between $m_{a}$ and $m_{0}+k$ such that $x_{r}=\xi^{\prime}, x_{r+1}=\xi$ and so there is just one $\mu$ greater than $m_{\sigma}$ (for the least $\mu, \mu_{0}$, exceeds $m_{0}$ ). Thus $\tau=\sigma$, which completes the proof.

In the same way we can show that each pair of values $\eta, \eta^{\prime}$ is takcu an even number of times by consecutive $y$ 's.

It follows from theorem 5 that in a simple closed $p$-curve there are an even number of boundary levels in each horizontal strip, and an even number in each vertical strip. If $x_{l}, x_{g}$ are the least and greatest values of $x_{r}, 0 \leqslant r \leqslant k$, and $y_{t}, y_{g}$ the least and greatest values of $y_{r}$, $0 \leqslant r \leqslant \hbar$, and if $2^{p} X_{s}, 0 \leqslant s \leqslant \sigma$ are the integers from $2^{p} x_{l}$ to $2^{p} x_{g}$ inclusive, and $2^{p} Y_{t}, 0 \leqslant t \leqslant \tau$, the integers from $2^{p} y_{l}$ to $2^{p} y_{y}$, and if, finally, $\eta_{r}^{s}$, $1 \leqslant r \leqslant 2 \mu_{s}$, are the boundary levels in the strip $X_{s}, X_{s+1}$ and $\xi_{r}^{t}, 1 \leqslant r \leqslant 2 v_{t}$, the boundary levels in the strip $\Gamma_{t}, Y_{t+1}$ then the cells of the network $F_{p}$ in all the rectangles $\left\langle X_{s}, X_{s+1}\right\rangle\left\langle\eta_{2 r-1}^{s}, \eta_{2 r}^{s}\right\rangle, 1 \leqslant r \leqslant \mu_{s}, 0 \leqslant s \leqslant \sigma-1$, are called the interior $p_{x}$-cells of the curve, and the cells of the network $F_{p}$ in all the rectangles $\left\langle\xi_{2 r-1}^{t}, \xi_{2 r}^{t}\right\rangle,\left\langle Y_{t}, Y_{t+1}\right\rangle, 1 \leqslant r \leqslant v_{t}, 0 \leqslant \tau \leqslant r-1$ are called the interior $p_{y}$-cells of the curve. A cell of the network $F_{p}$, in a vertical strip, which is not an interior $p_{x}$-cell is called an exterior $p_{x}$-cell, and a cell in a horizontal strip which is not an interior $p_{y}$-cell is called an exterior $p_{y}$-oell.

6. Linked boundary levels. $\left(x_{r}, y_{r}\right), 0 \leqslant r \leqslant k$, is a simple closed curve on a network $F_{p}$, where $x_{r}, y_{r}$ are periodic with period $k$.

A level $\alpha$ (of the closed curve) in the strip $\xi^{*}, \xi$ is said to be linked along $\xi$ to the level $\beta$ in the strip $\xi$, $\xi^{\prime}$ if eithes $\beta=\alpha$ or $\beta \geqslant \alpha$ and for some $\mu, \nu>1$

$$
x_{\mu}=\xi^{*}, \quad x_{\mu+p+1}=\xi^{\prime}, \quad x_{r}=\xi, \quad \mu+1 \leqslant r \leqslant \mu+\nu,
$$

and $y_{\mu}=\alpha, y_{\mu+\nu+1}=\beta$ and $2^{p} y_{r}, \mu+1 \leqslant r \leqslant \mu+\nu$, are the integers from $2^{p} a$ to $2^{p} \beta$ inclusive.

Two levels $\alpha, \beta$ of the same strip $\xi^{*}, \xi$ or $\xi, \xi^{*}$ are said to be linked along $\xi$ if $y_{\mu}=\alpha, y_{\mu+\nu+1}=\beta$ and $2^{p} y_{r}, \mu+1 \leqslant r \leqslant \mu+\nu$, are the integers from $2^{p} \alpha$ to $2^{p} \beta$ inclusive, and $x_{r}=\xi, \mu+1 \leqslant r \leqslant \mu+\nu$, and either $x_{\mu}$ $=x_{\mu+\nu+1}=\xi^{*}$ or $x_{\mu}=x_{\mu+v+1}=\xi^{\prime}$.

6.1. If a level $\alpha$ is linked to a level $\beta$ along $\xi$ then a is not linked to another level alang $\xi$; for, in a simple curve, there cannot be two values of $r, 0 \leqslant r \leqslant 7$, for which $y_{r}=y_{r+1}=\alpha$ and either $x_{r}=\xi^{*}, x_{r+1}=\xi$ or $x_{r}=\xi^{\prime}$, $x_{r+1}=\xi$.

6.2. If $\alpha, \beta$ are consecutive boundary levels in either of the strips $\xi^{*}, \xi ; \xi, \xi^{\prime}$ and if $\alpha \leqslant \eta<\eta^{\prime} \leqslant \beta$, then if for some $\mu, x_{\mu}=x_{\mu+1}=\xi$ and $y_{\mu}=\eta$, $y_{\mu+1}=\eta^{\prime}$ (or $y_{\mu}=\eta^{\prime}, y_{\mu+1}=\eta$ ) the levets $\alpha, \beta$ are linked along $\xi$.

Let $2^{p} \varrho_{s}, 0 \leqslant s \leqslant \sigma$, be the integers from $2^{p} a$ to $2^{p} \beta$ inclusive, where $\varrho_{t}=\eta, \varrho_{t+1}=\eta^{\prime}$, say, $0 \leqslant t<\sigma$. If $\alpha=\eta, \beta=\eta^{\prime}$ there is nothing to prove; hence we may suppose $\mu \geqslant 2$ so that for $1 \leqslant s \leqslant \sigma-1$, 淉 not a boundary level in either of the strips $\xi^{*}, \xi ; \xi, \xi^{\prime}$.

Accordingly $x_{\mu+2}=\xi, y_{\mu+2}=\varrho_{t+2}$ and, by induction, $x_{n}=\xi, y_{n}=\varrho_{n+t-1}$ for $\mu \leqslant n \leqslant \mu+\sigma-t$. Similarly $x_{m}=\xi, y_{m}=\varrho_{m+t-\mu}$ for $\mu-t \leqslant m \leqslant \mu-1$, which completes the proof.

6.21. It follows from 6.2 that if the consecutive boundary levels is, $\beta$ are not linked along $\xi$ then there is no value of $\mu, 0 \leqslant \mu \leqslant k$, for which $x_{\mu}$ $=x_{\mu+1}=\xi$ and $2^{p} y_{\mu}, 2^{p} y_{\mu+1}$ are conseeutive integers between $2^{p} \alpha$ and $2^{p} \beta$ inolusive.

6.3. If $\alpha, \beta, \gamma(\alpha<\beta<\gamma)$ are consecutive boundary levels in either of the strips $\xi^{*}, \xi$ or $\xi$, $\xi^{\prime}$ then either $\beta$ is a level in both strips, or $\beta$ is linked along $\xi$ either to $a$ or to $\gamma$. 
For if $\beta$ is not a level in both strips and if $y_{1}=y_{p+1}=\beta$ and one of $x_{\mu}, x_{\mu+1}$ is $\xi$, then two cases arise:

(a) $x_{\mu+1}=\xi$, then $x_{\mu+2}=x_{\mu+1}$;

(b) $x_{\mu}=\xi$, then $x_{\mu-1}=x_{\mu}$.

In case (a) either $y_{\mu+2}=\beta^{\prime}$ or $y_{\mu+2}=\beta^{*}$; if the former then $\beta$ is linked along $\xi$ to $\gamma$, and if the latter, $\beta$ is linked to $\alpha$, by 6.2 . The result follows in the same way in case (b). We observe that if $\gamma$ is the greatest boundary level in either strip, and if $\gamma$ is a level in only one of the two strips, and there is no greater boundary level in the other strip, then the foregoing considerations show that $\gamma$ is necessarily linked to $\beta$ along $\xi$. If $\gamma$ is a level in both strips then, by definition, $\gamma$ is linked along $\xi$.

6.4. We take for granted the definitions and theorems on linked levels in horizontal strips corresponding to 6-6.3 above.

6.5. If $\left\langle\xi^{*}, \xi\right\rangle\left\langle\eta, \eta^{\prime}\right\rangle$ and $\left\langle\xi, \xi^{\prime}\right\rangle\left\langle\eta, \eta^{\prime}\right\rangle$ are interior $p_{x}$ cells of a simple closed contour $\left(x_{r}, y_{r}\right), 0 \leqslant r \leqslant k$, then $\xi$ is not a boundary level in the sirnp $\eta, \eta^{\prime}$.

There are an odd number of levels in the strip $\xi^{*}, \xi$ which are not less than $\eta^{\prime}$, and an odd number in the strip $\xi$, $\xi^{\prime}$. Hence there are an even number of levels which lie in one or other of the two strips, and which are not less than $\eta^{\prime}$ (counting twice a value of $y_{r}$ which is a level in both strips). Let these levels, in decreasing order of magnitude be $h_{r}$, $1 \leqslant r \leqslant 2 n$, (where for some values of $r, h_{r}$ may equal $h_{r+1}$ ). By $6.3, h_{1}$ is linked to $h_{2}, h_{3}$ to $h_{4}$ and hence by induction, $h_{2 r-1}$ is linked to $h_{2 r}$ for $1 \leqslant r \leqslant n$. If $h_{2 n+1}$ is the first level in either strip which is less than $\eta^{\prime}$ (and so not greater than $\eta$ ) then $h_{2 n}$, being linked to $h_{2 n-1}$, is not linked. to $h_{2 n+1}$, and so by 6.21 there is no $\mu, 0 \leqslant \mu<k$, such that $x_{\mu}=x_{\mu+1}=\xi$ and $y_{\mu}=\eta, y_{\mu+1}=\eta^{\prime}$ (or $y_{\mu+1}=\eta, y_{\mu}=\eta^{\prime}$ ) which proves that $\xi$ is not a boundary level in the horizontal strip $\eta, \eta^{\prime}$.

6.51. If one of the $p_{x}$-cells $\left\langle\xi^{*}, \xi\right\rangle\left\langle\eta, \eta^{\prime}\right\rangle,\left\langle\xi, \xi^{\prime}\right\rangle\left\langle\eta, \eta^{\prime}\right\rangle$ is interior, and one exterior, then $\xi$ is a boundary level in the strip $\eta, \eta^{\prime}$, and conversely.

Proof similar to 6.5 .

7. ThEOREM 7. In a simple closed curvo $\left(x_{r}, y_{r}\right), 0 \leqslant r \leqslant \pi$, the interior

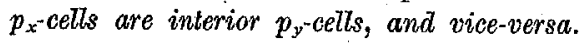

Iuet the boundary levels in the strip $\eta, \eta^{\prime}$ be $\xi_{s}, 1 \leqslant s \leqslant 2 \mu$, where $\xi_{s+1}>\xi_{s}, 1 \leqslant s \leqslant 2 \mu-1$.

If $\sigma$ is less than $x_{r}, 0 \leqslant r \leqslant k$, then, by 6.51 , there are no interior $p_{x}$-cells in the rectangle $\left\langle\sigma, \xi_{1}\right\rangle\left\langle\eta, \eta^{\prime}\right\rangle$, and therefore, by 6.51 , all the interior $p_{x}$-cells which lie in the strip $\eta, \eta^{\prime}$ lie in the rectangles $\left\langle\xi_{2 r-1}, \xi_{2 n}\right\rangle$ $\left\langle\eta, \eta^{\prime}\right\rangle$, and are therefore $p_{y^{-}}$-cells. Similanly the interior $p_{y}$-cells aro $p_{x^{-}}$-cells.

In view of Theorem 7 we now drop the suffix and refer to interior

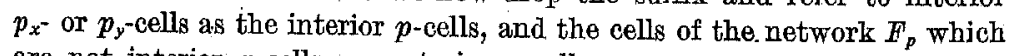
are not interior $p$-cells, as exterior $p$-cells.
8. For any $s, 0 \leqslant s \leqslant k-1$, the simple curves $\left(x_{r}, y_{r}\right), s \leqslant r \leqslant s+1$, joining the points $\left(x_{s}, y_{s}\right),\left(x_{s+1}, y_{s+1}\right)$, are called the boundary lines of the simple closed curve $\left(x_{r}, y_{r}\right), 0 \leqslant r \leqslant k$; for any assigned valne of $r$ between 0 and $z_{0}$ inclusive, the point $\left(x_{r}, y_{r}\right)$ is called a boundary p-point or vertex of the simple closed curve.

9. Let $2^{p} a_{r}, 0 \leqslant r \leqslant \mu$, be the integers from $2^{p} a$ to $2^{p} b$ inclusive, and $2^{p} \gamma_{r}, 0 \leqslant r \leqslant \nu$, the integers from $2^{p} e$ to $2^{p} d$ inclusive, then if

$$
\begin{array}{llr}
x_{r}=a_{r}, & y_{r}=c, & 0 \leqslant r \leqslant \mu, \\
x_{r}=b, & y_{r}=y_{r-\mu}, & \mu \leqslant r \leqslant \mu+\nu, \\
x_{r}=a_{2 \mu+\nu-r}, & y_{r}=d, & \mu+v \leqslant r \leqslant 2 \mu+v, \\
x_{r}=a, & y_{r}=\gamma_{2 \mu+2 p-r}, & 2 \mu+v \leqslant r \leqslant 2(\mu+\nu), \\
x_{r}^{*}=x_{2 \mu+2,-r}, & y_{r}^{*}=x_{2 \mu+2 r-r}, & 0 \leqslant r \leqslant 2(\mu+\nu),
\end{array}
$$

and

then the simple elosed curve $\left(x_{r}^{*}, y_{r}^{*}\right), 0 \leqslant r \leqslant 2(\mu+v)$, (or an equivalent $p$-curve) is called the clockwise $p$-path round the rectangle $\langle a, b\rangle\langle c, d\rangle$, and $\left(x_{r}, y_{r}\right), 0 \leqslant r \leqslant 2(\mu+\nu)$, is called the anticlockwise $p$-path.

The sides of a $p$-path round a $p$-cell are called the sides of the cell. An interior cell of a simple closed curve, which has a side in common with the curve, is called an interior boundary cell.

9.1. For any lattice point $(\xi, \eta)$ of a network $F_{p}$ we define:

and

$$
\begin{aligned}
& \left.\begin{array}{ll}
x_{r}^{s}=\xi, & r=0,3,4 \\
x_{r}^{s}=\xi^{\prime}, & r=1,2 \\
=y_{1}^{s}=y_{4}^{s}, & y_{3}^{s}=y_{3}^{s},
\end{array}\right\} 1 \leqslant s<4, \\
& y_{0}^{s}=\eta, \quad s=1,2, \quad y_{2}^{s}=\eta^{\prime}, \quad s=1, \\
& y_{0}^{s}=\eta^{\prime}, \quad s=3, \quad y_{2}^{s}=\eta, \quad s=3,4 \text {, } \\
& y_{0}^{s}=\eta^{*}, \quad s=4, \quad y_{2}^{s}=\eta^{*}, \quad s=2 .
\end{aligned}
$$

It is readily verified that the simple closed $p$-curves $\left(x_{r}^{s}, y_{r}^{s}\right), 0 \leqslant r \leqslant 4$, are clockwise for $s=2,3$ and anticlockwise for $s=1,4$. In the curves given by $s=1,2$ the point $(\xi, \eta)$ precedes the point $\left(\xi^{\prime}, \eta\right)$, and in the curves $s=3,4$ the point $\left(\xi^{\prime}, \eta\right)$ precedes $(\xi, \eta)$.

Thus of the four curves there is one clockwise and one anticlockwise curve in which $(\xi, \eta)$ precedes $\left(\xi^{\prime}, \eta\right)$, and one clockwise and one anticlockwise curve in which $\left(\xi^{\prime}, \eta\right)$ precedes $(\xi, \eta)$.

9.11. Let $\eta$ be a boundary level, in the strip $\xi$, $\xi^{\prime}$, of a simple closed curve $\Gamma$; then of the four curves $\left(x_{r}^{s}, y_{r}^{s}\right), 0 \leqslant r \leqslant 4$, either those with $s=1,3$ or those with $s=2,4$ are paths round the interior boundary cell with 
vertices $(\xi, \eta),\left(\xi^{\prime}, \eta\right)$. In either case there is only one curve in which the order of the points $(\xi, \eta),\left(\xi^{\prime}, \eta\right)$ is the same as in $\Gamma$. Similarly there is only one path round an interior boundar'y cell with vertices $(\xi, \eta),\left(\xi^{\prime}, \eta\right)$ in which the order of these points is the same as in $I$. Thus with each boundary line of $\Gamma$ we have associated a unique path, round an interior boundary cell, and this path is said to be described in the same sense as $I$. We shall show that all the paths described round interior boundary cells of $T$, and described in the same sense as $\Gamma$, are either all clockwise, or all anticlockmise. In the former case the curve $I$ is said to be clockwise, and in the latter, anticlockwise.

9.12. Let the path round the interior boundary cell of $\Gamma$, with vertices $(\xi, \eta),\left(\xi^{\prime}, \eta\right)$, be the anticlockwise curve $\left(x_{r}^{2}, y_{r}^{1}\right), 0 \leqslant r \leqslant 4$, and consider the successor, in $\Gamma$, of the points $(\xi, \eta),\left(\xi^{\prime}, \eta\right)$.

If the successor is $\left(\xi^{\prime \prime}, \eta\right)$ then $\xi^{\prime}$ is not a boundary level in the strij) $\eta, \eta^{\prime}$ and so $\left\langle\xi^{\prime}, \xi^{\prime \prime}\right\rangle\left\langle\eta, \eta^{\prime}\right\rangle$ is an interior boundary cell; thus the patl associated with the side of $T$ joining the points $\left(\xi^{\prime}, \eta\right),\left(\xi^{\prime \prime}, \eta\right)$ is the anticlookwise curve

$$
\left(\xi^{\prime}, \eta\right), \quad\left(\xi^{\prime \prime}, \eta\right), \quad\left(\xi^{\prime \prime}, \eta^{\prime}\right), \quad\left(\xi^{\prime}, \eta^{\prime}\right), \quad\left(\xi^{\prime}, \eta\right),
$$

(i. e. the curve $\left(x_{r}^{1}, y_{r}^{1}\right), 0 \leqslant r \leqslant 4$, with $\xi$ replaced by $\xi^{\prime}$ and so $\xi^{\prime}$ replaced by $\left.\xi^{\prime \prime}\right)$.

If $\left(\xi^{\prime}, \eta^{\prime}\right)$ is the successor, then $\xi^{\prime}$ is a boundary level in the strip $\eta, \eta^{\prime}$ and so $\left\langle\xi, \xi^{\prime}\right\rangle\left\langle\eta, \eta^{\prime}\right\rangle$ is the interior cell associated with the side of $I$ joining the points $\left(\xi^{\prime}, \eta\right),\left(\xi^{\prime}, \eta^{\prime}\right)$, and the path associated with this side is the anticlockwise curve $\left(x_{r}^{1}, y_{r}^{1}\right), 0 \leqslant r \leqslant 4$.

If $\left(\xi^{*}, \eta^{*}\right)$ is the successor, then, since $\eta$ is a boundary level in the strip $\left(\xi, \xi^{\prime}\right),\left\langle\xi, \xi^{\prime}\right\rangle\left\langle\eta^{*}, \eta\right\rangle$ is an exterior cell and so $\left\langle\xi^{\prime}, \xi^{\prime \prime}\right\rangle\left\langle\eta^{*}, \eta\right\rangle$ is the interior cell associated with the side joining the points $\left(\xi^{\prime}, \eta\right),\left(\xi^{\prime}, \eta^{*}\right)$ and the path associated with this side is the anticlockiswise curve $\left(x_{r}^{4}, y_{r}^{a}\right)$, $\leqslant r \leqslant 4$, with $\xi^{\prime}$ replacing $\xi$ and $\xi^{\prime \prime}$ replacing $\xi^{\prime}$.

The same analysis may be applied to a consideration of the successor of the points $(\xi, \eta),\left(\xi, \eta^{\prime}\right)$. Thus if the path round one interior boundary cell, described in the same sense as the curve $\Gamma$, is anticlockwise, then interior boundary, described in the same sense as $\Gamma$, round any other interior boundary cell. And if one is clockwise, then all are clockwise.

10. If $\Gamma_{p+1}$ is the closed curve on a network $F_{p+1}$ which is equivalent to a curve $\Gamma_{p}$ on a network $F_{p}$, then the interior $(p+1)$-cells of $\Gamma_{p+1}$ are the $(p+1)$-cells of the interior $p$-cells of $\Gamma_{p}$. For if $y_{r}^{p}$ is a boundary level in the strip $x_{r}^{p}, x_{r+1}^{p}$ then $y_{2 r}^{p+1}=y_{r}^{p}$ is a boundary level in each of the strips $x_{2 r}^{p+1}, x_{2+1}^{p+1} ; x_{2 r+1}^{p+1}, x_{2+2}^{p+1}$. Similarly for boundary levels in horizontal
strips.
11. $\Gamma$ and $\gamma$ are simple closed curves on a netrork $F_{p}$. If all the interior cells of $\gamma$ are interior cells of $\Gamma$, and all the interior cells of $T$ which have a vertex in common. with $I, i, e$. the interior boundary cells of $\Gamma$, are exterior to $\gamma$, then $\gamma$ is said to be completely contained in $\Gamma$.

$\gamma$ and $\Gamma$ are said to be completely exterior to each other if no interior cell of one is interior to the other and no boundary $p$-point of one is a boundary $p$-point of the other.

11.1. If $\gamma$ is completely contained in $\Gamma$, all the cells exterior to $\gamma$, with a verten in common with $\gamma$, are interior to $\Gamma$.

Let $\left\langle\xi, \xi^{\prime}\right\rangle\left\langle\eta, \eta^{\prime}\right\rangle$ be a cell exterior to $\gamma$, and $(\xi, \eta)$ a boundary $p$-point of $\gamma$. If $\xi$ is a boundary level of $\gamma$ in the strip $\eta, \eta^{\prime}$ then the cell $\left\langle\xi^{*}, \xi\right\rangle$ $\left\langle\eta, \eta^{\prime}\right\rangle$ is an interior cell of $\gamma$, and so of $\Gamma$; hence neither $(\xi, \eta)$ nor $\left\langle\xi, \eta^{\prime}\right)$ are boundary points of $T$ and so $\xi$ is not a boundary level of $T$ in $\eta, \eta^{\prime}$. Therefore $\left\langle\xi, \xi^{\prime}\right\rangle\left\langle\eta, \eta^{\prime}\right\rangle$ is interior to $I$. The corresponding result holds if $\eta$ is a boundary level of $\gamma$ in the strip $\xi, \xi^{\prime}$.

If $\xi$ is not a boundary level of $\gamma$ in $\eta, \eta^{\prime}$ and $\eta$ is not a boundary level in $\xi, \xi^{\prime}$, then $\left\langle\xi, \xi^{\prime}\right\rangle\left\langle\eta^{*}, \eta\right\rangle$ is exterior to $\gamma$; since $(\xi, \eta)$ is necessarily contained between $\left(\xi^{*}, \eta\right)$ and $\left(\xi, \eta^{*}\right)$, in $\gamma$, therefore $\left\langle\xi^{*}, \xi\right\rangle\left\langle\eta^{*}, \eta\right\rangle$ is interior to $\gamma$, and so interior to $\Gamma$. Hence $(\xi, \eta)$ is not a boundary point of $\Gamma$, and so $\xi$ is not a boundary level of $\Gamma$ in the strip $\left(\eta^{*}, \eta\right)$ and $\eta$ is not a boundary level of $\Gamma$ in $\xi, \xi^{\prime}$; accordingly both $\left\langle\xi, \xi^{\prime}\right\rangle\left\langle\eta^{*}, \eta\right\rangle$ and $\left\langle\xi, \xi^{\prime}\right\rangle\left\langle\eta, \eta^{\prime}\right\rangle$ are interior cells of $\Gamma$.

11.2. If $\gamma_{1}, \gamma_{2}, \gamma_{3}$ are simple closed curves, and if $\gamma_{1}$ is completely contained in $\gamma_{2}$ and $\gamma_{2}$ is completely contained in $\gamma_{3}$, then $\gamma_{1}$ is completely contained in $\gamma_{3}$.

For the interior cells of $\gamma_{1}$ are interior cells of $\gamma_{2}$, and the interior cells of $\gamma_{2}$ are interior cells of $\gamma_{3}$, so that the interior cells of $\gamma_{1}$ are interior to $\gamma_{3}$. Moreover, any cell which has a vertex in common with $\gamma_{3}$, is exterior to $\gamma_{2}$ and so exterior to $\gamma_{1}$.

11.3. A vertex of an interior $p$-cell of a simple closed curve which is not also a vertex of the curve, is called an interior p-point of the curve. A vertex of an exterior $p$-cell which is not a vertex of the curve, is called an exterior p-point.

If $L$ is an interior and $M$ an exterior p-point of a simple closed curve $\gamma$, then any simple p-eurve joining $I$ to $M$ has a verter in common with $\gamma$.

Let $k$ be a simple $p$-curve from $L$ to $M I$. There are boundary $p$-points of $t$ which are exterior to $\gamma(e . g . M)$; let $\left(a_{r+1}, b_{r+1}\right)$ be the first point of $z$ which is not an interior $p$-point of $\gamma$, so that $\left(a_{r}, b_{r}\right)$ is an interior $p$-point. Of the two cells with vertices $\left(a_{r}, b_{r}\right),\left(a_{r+1}, b_{r+1}\right)$ one at least is an interior $p$-cell, and so $\left(a_{r+1}, b_{r+1}\right)$ is a boundary $p$-point of $\gamma$. 
12. $\Gamma$ is the simple closed curve $\left(x_{r}^{p}, y_{r}^{p}\right), 0 \leqslant r \leqslant k$, on a network $F_{p}$, and $\gamma$ is a simple closed $p$-ourve $\left(a_{r}^{p}, b_{r}^{p}\right), 0 \leqslant r \leqslant \lambda$, such that each point $\left(a_{r}^{p}, b_{r}^{p}\right)$, $0 \leqslant r \leqslant \lambda$, is an interior point of $\Gamma$. Then $\gamma$ is completely contained in $\Gamma$.

Let $b_{\nu}^{p}$ be a boundary level of $\gamma$ in the strip $\left(a_{v}^{p}, a_{p-1-1}^{p}\right)$ with $b_{v}^{p}=b_{p+1}^{p}$; we may without loss of generality suppose that $a_{v+1}^{p}>a_{\nu}^{p}$. Since $\left(a_{v}^{p}, b_{v}^{p}\right)$ and $\left(a_{v+1}^{p}, b_{p+1}^{p}\right)$ are interior points of $\Gamma, b_{\nu}^{p}$ is contained between consecutive boundary levels of $\Gamma$ in the strip $\left(a_{\mu}^{p}, a_{p+1}^{p}\right)$, say $\eta_{1}, \eta_{2}$ where $\eta_{1}<\eta_{2}$, and let

$$
\begin{array}{llll}
x_{Q}=a_{p}^{p}, & x_{Q+1}=a_{\nu+1}^{p}, & x_{\sigma}=a_{\nu+1}^{p}, & x_{\sigma+1}=a_{\nu}^{p}, \\
y_{Q}=\eta_{1}, & y_{\mathrm{Q}+1}=\eta_{1}, & y_{\sigma}=\eta_{2}, & y_{\sigma+1}=\eta_{2} .
\end{array}
$$

We denote by $2^{p+1} e_{r}, 0 \leqslant r \leqslant h$, the integers from $2^{p} \eta_{2}$ to $2^{p} \eta_{\mathrm{R}}$ respect ively, and by $D$ the simple closed $(p+1)$-curve $\left(X_{r}, Y_{r}\right)$ where

and

$$
\begin{array}{lll}
X_{r}=x_{r+2+1}^{p+1}, & Y_{r}=y_{r+2}^{p+1}, & 0 \leqslant r \\
X_{r}=x_{2_{e}+1}^{p+1}, & Y_{r}=e_{2(\alpha-\rho)+h-r}, & 2(\sigma-\varrho)+1 \leqslant r \\
& x_{2 r}^{p+1}=x_{r}^{p}, \quad x_{2 r+1}^{p+1}=\frac{1}{2}\left(x_{r}^{n}+x_{r+1}^{p}\right), \\
& y_{2 r}^{p+1}=y_{r}^{p}, \quad y_{2 r+1}^{p+1}=\frac{1}{2}\left(y_{r}^{p}+y_{r+1}^{p}\right),
\end{array}
$$$$
0 \leqslant r \leqslant 2(\sigma-\varrho),
$$$$
2(\sigma-\varrho)+1 \leqslant \eta \leqslant 2(\sigma-\varrho)+h,
$$

(so that $\left(x_{r}^{p+1}, y_{r}^{p+1}\right), 0 \leqslant r \leqslant 2 k$, is equivalent to $\Gamma$ ).

Further, $L$ is the simple $(p+1)$-curve $\left(a_{r}^{p+1}, b_{r}^{p+1}\right), 2(\nu+1) \leqslant r \leqslant 2(\nu+\lambda)$, joining the points $\left(a_{v}^{p}, b_{v}^{p}\right),\left(a_{v+1}^{p}, b_{v+1}^{p}\right)$, where $\left(a_{r}^{p+1}, b_{r}^{p+1}\right), 0 \leqslant r \leqslant 2 \lambda$, is equivalent to $\left(a_{r}^{p}, b_{r}^{p}\right), 0 \leqslant r \leqslant \lambda$, and both $a_{r}^{p+1}, b_{r}^{p+1}$ are periodic with period $2 \lambda$.

The only boundary level of $D$, between $a_{v}^{p}$ and $a_{v+1}^{p}$, in any of the strips $e_{r}, e_{r+1}, 0 \leqslant r \leqslant h-1$, is $X_{0}$, and so one of the points $\left(a_{p}^{p}, b_{v}^{p}\right)$, $\left(a_{y+1}^{p}, b_{v+1}^{p}\right)$ is interior to $D$, and the other exterior to $D$. Hence by 11.3 , $L$ and $D$ have a boundary $(p+1)$-point in common. It may be shown that $T$ and $\gamma$ have no point in common, and so the common points of $L$ and $D$ are $\left(X_{0}, e_{r}\right)$ for some values of $r$. Let the common points be $\left(a_{r}^{p+1}, b_{r}^{p+1}\right), r=2(\nu+1)+r_{m}, 1 \leqslant m \leqslant n$, where $r_{m+1}>r_{m}$ and $r_{n}<2 \lambda$. The relation of $\left(a_{r}^{p+1}, b_{r}^{p+1}\right)$ to $\left(a_{r}^{p}, b_{r}^{p}\right)$ shows that, for $r=2(v+1)+r_{m}, 1 \leqslant m \leqslant n$, $b_{r}^{p+1}$ is a boundary level of $\gamma$ in the strip $a_{v}^{p}, b_{v+1}^{p}$, and a boundary level of $L$ in each of the strips $a_{2 v}^{p+1}, a_{2 v+1}^{p+1}$ and $a_{2 p+1}^{p+1}, a_{2 p+2}^{p+1}$. It follows that for $r=2(\nu+1)+r_{m}+(-1)^{m}, 1 \leqslant m \leqslant n$, the points $\left(a_{r}^{p+1}, b_{r}^{p+1}\right)$ lie on the same side of $D$ as $a_{r+1}^{p}$, and those for which $r=2(\nu+1)+r_{m}-(-1)^{m}, 1 \leqslant m \leqslant n$, lie on the same side as $a_{y}^{p}$; but $2(y+1)+r_{n}$ is the greatest value of $r$ (below $2(v+\lambda)$ ) for which $\left(a_{r}^{p+1}, b_{r}^{p+1}\right)$ is a common point of $L$ and $D$, and so $r_{n}+(-1)^{n}<r_{n}-(-1)^{n}$, which proves that $n$ is odd. Since $b^{n}$ itself is also a boundary level of $\gamma$ in the strip $a_{y}^{p}, a_{r+1}^{p}$, it follows that there are an even number of boundary levels of $\gamma$ in the strip $a_{v}^{p}, a_{\nu+1}^{p}$ which lie between the consecutive boundary levels of $\Gamma, \eta_{1}$ and $\eta_{2}$.

Thus between any two consecutive boundary levels of $\Gamma$, in a strip $\xi, \xi^{\prime}$ lie an even number of boundary levels of $\gamma$, (and no boundary level of $\gamma$ lies outside $\Gamma$ since the vertices of $\gamma$ are interior points of $\Gamma$ ) so that, if $f_{r}, 1 \leqslant r \leqslant 2 i$, are the boundary levels of $\Gamma$ in $\xi, \xi^{\prime}$ (in increasing order of magnitude) then the interior cells of $\gamma$ in this strip lie between $f_{2 r-1}$ and $f_{2 r}, 1 \leqslant r \leqslant i$, and are therefore all interior cells of $\Gamma$, and the boundary cells of $T$ are exterior cells of $\gamma$. Thus $\gamma$ is completely contained inside $\Gamma$.

\section{The $p$-curve of a relatively continuous function.}

13.1. A rational recursive function (see [1]) $f(n, x)$ is convergent in $n$, and continnous in $x$, relative to $n$, (op. cit., p. 174) in the interval $\langle a, b\rangle$, if there are recursive functions $N(k, x), a^{k}(r), \beta(k), \sigma(k, r)$ and $C(x, y, k)$ such that, for all positive integers $k$,

$$
|f(n, x)-f(N(k, \infty), x)|<1 / 2^{k}
$$

for all integers $n$ not less than $N(k, x)$, and all rational $x$ in $\langle a, b\rangle$, and, for $0 \leqslant r \leqslant \beta(k)$,

$$
\left|f(n, x)-f\left(n, a^{k}(r)\right)\right|<1 / 2^{k}
$$

for all $x$ satisfying $a^{k(r)} \leqslant x \leqslant a^{k}(r+1)$, and $n \geqslant C\left(x, a^{k}(r), k\right)$, where $a^{k}(0)=a$, $a^{k}(\beta(k)+1)=b$, and $a^{k}(r)<a^{k}(r+1), 0 \leqslant r \leqslant \beta(k)$, and $a^{k+1}(r)=a^{k}(\sigma(k, r))$.

13.2. $f(n, x)$ is convergent in $n$, and continuous relative to $n$, in $\langle a, b\rangle$; then each of the differences

$$
\begin{aligned}
& \left|f\left(n, a^{k+2}(r)\right)-f\left(N\left(k+2, a^{k+2}(r)\right), a^{k+2}(r)\right)\right| \\
& \left|f\left(n, a^{k+2}(r+1)\right)-f\left(N\left(k+2, a^{k+2}(r+1)\right), a^{k+2}(r+1)\right)\right| \\
& \left|f\left(n, a^{k+2}(r+1)\right)-f\left(n, a^{k+z}(r)\right)\right|
\end{aligned}
$$

is less than $1 / 2^{k+2}$, whence

$$
\left|f\left(N\left(k+2, a^{k+2}(r+1)\right), a^{k+2}(r+1)\right)-f\left(N\left(k+2, a^{k+2}(r)\right), a^{k+2}(r)\right)\right|<3 / 2^{k+2}
$$

and so, if

$$
f_{k}(r)=\left[2^{k} f\left(N\left(k+2, a^{k+2}(r)\right), a^{k+2}(r)\right)\right] / 2^{k}
$$

(where $[x]$ denotes the greatest integer not exceeding $x$, if $x$ is non-negative, and $[x]=-[-x]$ if $x$ is negative) then

$$
\left|f_{k}(r+1)-f_{k}(r)\right| \leqslant 1 / 2^{k}
$$

Fundamenta Mathematicae. T. XLIII 
and so the integers $2^{k} f_{k}(r)$ are equal or consecutive for consecutive valucs of $r . f_{k}(r)$ is called the lacing of the function $f(n, x)$; the lacing deyends, of course, upon the subdivision $a^{k}(r)$.

13.3. $f(n, x)$ and $g(n, x)$ are both convergent in $n$, and continuous relative to $n$, in $\langle a, b\rangle$. By combining, the subdivisions of $(a, b)$ associated with $f(n, x)$ and $g(n, \infty)$ respectively we may form lacings of these functions, $f_{k}(r)$ and $g_{k}(r)$, on a common subdivision $a^{k}(r), 0 \leqslant r \leqslant \beta(k)+-1$, say.

Let

$$
\begin{array}{lll}
\Theta(0)=0, & \Theta(r+1)=2^{p}\left\{f_{p}(r+1)-f_{p}(r)\right\}, & 0 \leqslant r \leqslant \beta(p), \\
\varphi(0)=0, & \varphi(r+1)=2^{p}\left\{g_{p}(r+1)-g_{p}(r)\right\},
\end{array}
$$

so that $\Theta(r)$ and $\varphi(r)$ take only the values 0 , \pm .1.

Further, let $r_{0}=0$, and let $r_{r+1}$ be the least integer greater tilon $r_{n}$, if any, such that $\left|\Theta\left(r_{n+1}\right)\right|+\left|\varphi\left(r_{n+1}\right)\right|>0$; otherwise $r_{n+1}=r_{n} . \mu_{p}$ is the greatest positive integer, if any, such that $\mu_{p} \leqslant \beta(p)+1$ and $\gamma_{\mu_{p}}>\gamma_{\mu_{p}}-1$; otherwise $\mu_{p}=0$.

Hence if $f_{p}^{*}(i)=f_{p}\left(r_{i}\right)$ and $g_{p}^{*}(i)=g_{p}\left(r_{i}\right), 0 \leqslant i \leqslant \mu_{p}$, and

$$
\begin{array}{ll}
\Theta^{*}(0)=0, & \Theta^{*}(i+1)=2^{p}\left\{f_{p}^{*}(i+1)-f_{p}^{*}(i)\right\} \\
\varphi^{*}(0)=0, & \varphi^{*}(i+1)=2^{p}\left\{g_{p}^{*}(i+1)-g_{p}^{*}(i)\right\}
\end{array}
$$

then $\Theta^{*}(i), \varphi^{*}(i)$ take only the values $0, \pm 1$, and are not simultaneonsly zero for $i>0$.

Next, let $k_{1}+1, k_{2}+1, \ldots, k_{v}+1$ be the values of $i$, (if any) in increasing order of magnitude, where $\left|\Theta^{*}(i) \varphi^{*}(i)\right|>0$, then we define:

$$
\text { and }
$$$$
\left.\begin{array}{ll}
f^{p}\left(k_{r}+r\right)=f_{p}^{*}\left(k_{r}+1\right), & f^{p}(j)=f_{p}^{*}(j-s) \\
g^{p}\left(k_{r}+r\right)=g_{p}^{*}\left(k_{r}\right), & g^{p}(j)=g_{p}^{*}(j-s)
\end{array}\right\} \begin{aligned}
& 1 \leqslant r \leqslant v, \\
& k_{s}+s+1 \leqslant j \leqslant k_{s+1}+s \\
& 1 \leqslant s \leqslant v-1
\end{aligned}
$$

$$
\begin{array}{llrl}
f^{p}(j)=f_{p}^{*}(j), & g^{p}(j)=g_{p}^{*}(j), & 0 \leqslant j \leqslant k_{1}, \\
f^{p}(j)=f_{p}^{*}(j-\nu), & g^{p}(j)=g_{p}^{*}(j-\nu), & k_{p}+\nu+1 \leqslant j \leqslant \mu_{p}+\nu .
\end{array}
$$

If $\Theta^{*}(i) \varphi^{*}(i)=0$ for all $i, 0 \leqslant i \leqslant \mu_{p}$, then wo define

In either case

$$
f^{p}(i)=f_{p}^{*}(i), \quad g^{p}(i)=g_{p}^{*}(i), \quad 0 \leqslant i \leqslant \mu_{p} .
$$

and therefore

$$
\begin{gathered}
2^{p}\left\{\left|f^{p}(i+1)-f^{p}(i)\right|+\left|g^{p}(i+1)-g^{p}(i)\right|\right\}=1 \\
\left(f^{p}(r), g^{p}(r)\right), \quad 0 \leqslant r \leqslant \mu_{p}+v,
\end{gathered}
$$

(where $\nu$ is the number of values of $i$ for which $\left|\Theta^{*}(i) \varphi^{*}(i)\right|>0$ ) is a plane p-curve, which twe shall call the p-curve derived from $f(n, x), g(n, x)$.
Thus a pair of functions $f(n, x), g(n, x)$, each convergent in $n$, and continuons in $x$, relative to $n$, determine a sequence of curves, the $p$-curves derived from the pair, for all positive integral values of $p$.

14. If $f\left(r^{*}\right)$ is the lacing of a function $f(n, x)$ on a subdivision $a(r)$, $0 \leqslant r \leqslant \beta+1$, then $\sum_{r=0}^{\beta}|f(r+1)-f(r)|$ is called the relative nariation of $f(n, x)$ on the subdivision $a(r)$.

14.1. If $V^{T}, V^{2}$ are the relative variations of $f(n, x)$ on the subdivisions $a_{1}(r), \quad 0 \leqslant r \leqslant \beta_{1}+1 ; a_{2}(r), 0 \leqslant r \leqslant \beta_{2}+1$, and if there is a positive integer function $v_{p}$ snch that, for $k \geqslant 1$,

$$
\left|V^{1}-V^{2}\right|<1 / k
$$

for any subdivisions $a_{1}(r), a_{2}(r)$ satisfying

$$
\max _{\substack{0 \leqslant r \leqslant \beta_{1} \\ 0 \leqslant s \leqslant \beta_{2}}}\left\{\left(a_{1}(r+1)-a_{1}(r)\right),\left(a_{2}(s+1)-a_{2}(s)\right)\right\}<1 / v_{k},
$$

then (anticipating the follorring theorem) $f(n, x)$ is said to be of convergent variation relative to $n$.

14.2. If $f(n, \infty)$ is of convergent variation relative to $n$, and if $V_{p}$ is the relative variation on a subdivision $a^{p}(r), 0 \leqslant r \leqslant \beta(p)+1$, such that $\max \left\{a^{p}(r+1)-a^{p}(r)\right\} \rightarrow 0$, then $\nabla_{p}$ is convergent. $0 \leqslant r \leqslant \beta(p)$

For we can determine $p_{k}$ snch that $\max \left\{a^{p}(r+1)-a^{p}(r)\right\}<1 / v_{k}$ for $p \geqslant p_{k}$, and so if $q$ is any positive integer, then by 14.1,

$$
\left|V_{p+q}-V_{p}\right|<1 / k
$$

which proves that $V_{p}$ converges.

14.3. If $f(n, x), g(n, x)$ are of convergent variation relative to $n$, then the sequence of $p$-curves derived from the pair of functions is said to be rectifiable.

\section{Reference}

[1] R. I. Goodstein, Recursive function theary, Acta Math. 92 (1954), p. 171-180. UNIVERSITY COLLLEOE OF LEICESTER

Reģu par la Rédaction le 10.10.1954 